OPEN ACCESS

Edited by:

Ana Sandoval-Rodriguez, University of Guadalajara, Mexico

Reviewed by: Jaswinder Singh Maras, The Institute of Liver and Biliary

Sciences (ILBS), India Eira Cerda Reyes, HCM, Mexico

*Correspondence: Jian-Hong Zhong zhongjianhong@gxmu.edu.cn

${ }^{+}$These authors have contributed equally to this work

Specialty section: This article was submitted to Gastrointestinal Cancers: Hepato

Pancreatic Biliary Cancers, a section of the journal

Frontiers in Oncology

Received: 26 September 2021 Accepted: 29 December 2021

Published: 20 January 2022

Citation:

Liu L, Xie S, Teng Y-X, Deng Z-J, Chen K, Liu H-T, Huo R-R, Liang X-M, Guo $P$ - $P$, Yang $D-L, M a L$, Xiang $B-D$,

Li L-Q and Zhong J-H (2022) Outcomes of Liver Resection for

Metabolic Dysfunction-Associated

Fatty Liver Disease or Chronic Hepatitis B-Related HCC.

Front. Oncol. 11:783339. doi: 10.3389/fonc.2021.783339

\section{Outcomes of Liver Resection for Metabolic Dysfunction-Associated Fatty Liver Disease or Chronic Hepatitis B-Related HCC}

\author{
Lei Liu ${ }^{1 \dagger}$, Si Xie ${ }^{2+}$, Yu-Xian Teng ${ }^{2 \dagger}$, Zhu-Jian Deng ${ }^{2}, K_{\text {Kang Chen }}^{2}$, Hao-Tian Liu ${ }^{2}$, \\ Rong-Rui Huo ${ }^{2}$, Xiu-Mei Liang ${ }^{2}$, Ping-Ping Guo ${ }^{2}$, Da-Long Yang ${ }^{2}$, Liang $\mathrm{Ma}^{2}$, \\ Bang-De Xiang ${ }^{2}$, Le-Qun $\mathrm{Li}^{2}$ and Jian-Hong Zhong ${ }^{2 *}$

\begin{abstract}
Hepatobiliary Surgery Department, Fourth Affiliated Hospital of Guangxi Medical University, Liuzhou Workers Hospital, Liuzhou, China, ${ }^{2}$ Hepatobiliary Surgery Department, Guangxi Liver Cancer Diagnosis and Treatment Engineering and Technology Research Center, Guangxi Medical University Cancer Hospital, Nanning, China
\end{abstract}

Aims: This study aims to determine differences in severity of background liver disease at hepatocellular carcinoma (HCC) diagnosis and long-term survival outcomes among patients undergoing liver resection for HCC in the background of metabolic dysfunction-associated fatty liver disease (MAFLD) compared to chronic hepatitis $B$ (CHB) alone or concurrent $\mathrm{CHB}$ (CHB/MAFLD).

Methods: Patient demographics and comorbidities, clinicopathologic data, perioperative and long-term outcomes among patients who underwent liver resection for HCC were reviewed. Overall and recurrence-free survival were calculated with the Kaplan-Meier method, with the values compared using the log-rank test.

Results: From January 2014 to December 2018, 1325 patients underwent potential curative liver resection of HCC; 67 (5.0\%), 176 (13.3\%), and 1082 (81.7\%) patients had MAFLD alone, CHB concurrent with MAFLD, and CHB alone, respectively. At HCC diagnosis, fewer MAFLD patients had cirrhosis, alpha fetoprotein concentration $\geq 400 \mathrm{ng} /$ $\mathrm{mL}$, tumor size $\geq 5 \mathrm{~cm}$, mulinodular, microvascular invasion, receiving major hepatectomy, and receiving adjuvant transarterial chemoembolization. After a median follow-up of 47 months after liver resection, MAFLD (or MAFLD plus CHB/MAFLD) patients had significantly higher overall and recurrence-free survival than $\mathrm{CHB}$ patients before or after propensity score analysis (all $P<0.05$ ).

Conclusion: Patients with HCC in the setting of MAFLD have less-severe background liver disease at HCC diagnosis and better long-term survival after curative liver resection compared to counterparts with CHB/MAFLD or CHB.

Keywords: chronic hepatitis B, hepatocellular carcinoma (HCC), liver resection, metabolic dysfunction-associated fatty liver disease, overall survival 


\section{INTRODUCTION}

Hepatocellular carcinoma (HCC) is the sixth most common malignancy and the third leading cause of cancer-related death (1), with East Asia demonstrating the highest incidence of HCC worldwide (2). Past epidemiological data suggest that hepatitis B virus (HBV), hepatitis $\mathrm{C}$ virus ( $\mathrm{HCV}$ ), and alcohol consumption are three predominant causative factors of HCC. In recent decade, nonalcoholic fatty liver disease (NAFLD) is already a rapidly increasing risk factor of HCC in the USA, France and the UK (3, 4). The estimated annual incidence of HCC among patients with NAFLD is lower than that among those with HBV or HCV infection $(5,6)$. However, it is estimated that $25 \%$ of the global population have NAFLD, with the highest prevalence in high income regions (6). Namely, more worldwide people have NAFLD than other liver diseases, such as $\mathrm{HBV}$ or $\mathrm{HCV}$ infection, leading the necessary to analyze the prognoses of patients with NAFLD-related HCC.

NAFLD is defined as excess hepatic fat accumulation ( $>5 \%)$ after the exclusion of significant alcohol consumption or any other causes of steatosis, such as certain toxins and drugs (7-10). Metabolic dysfunction-associated fatty liver disease (MAFLD), formerly named NAFLD, is a chronic disease characterized by fat accumulation in the liver with an underlying metabolic dysregulation, for which there is no approved pharmacotherapy (10). Recently, several official guidelines and consensus provide simple and practical diagnostic criteria for the disease (10-12). MAFLD more closely implies the presence of type 2 diabetes mellitus, overweight/obesity, or metabolic dysregulation, contributing to better identification of individuals with this metabolic liver disease (13). More importantly, MAFLD may eventually progress to liver-related complications, including HCC. A populational cohort from Switzerland found the burden of NAFLD- and MAFLD-related HCCs significantly increased over the years, particularly in women (14).

Liver resection is one of the main curative treatments for patients with HCC $(15,16)$. However, HCC recurrence after liver resection is high. Systematic reviews with large sample size revealed that the 5-years recurrence-free survival are $37 \%, 25 \%$, and $18 \%$ in patients with early, intermediate, or advanced disease, respectively $(16,17)$. And the corresponding 5 -years overall survival are $67 \%$, $30 \%$, and $18 \%$, respectively $(16,17)$. Tumor stage and treatment measures are the most important factors affecting the prognosis of patients with HCC. However, our understanding of the association between etiological factors and the prognoses of patients with HCC after liver resection is limited. Furthermore, the contribution of MAFLD to the HCC burden according to clinical and tumor characteristics remain unclear. We therefore aimed to compare the clinical and tumor characteristics and the outcomes of patients with HBV- or MAFLD-related HCC after liver resection.

\section{PATIENTS AND METHODS}

\section{Study Design and Population}

This was a retrospective study. All patients with a diagnosis of HCC based on postoperative histopathology between 1 January 2014 and 31 December 2018 might be eligible for the study.
Consecutive patients with HCC were identified via the electronic medical records of Guangxi Medical University Cancer Hospital, Nanning, China, and the Fourth Affiliated Hospital of Guangxi Medical University, Liuzhou, China. Patients were selected based on the following eligibility criteria: (1) patients were without preoperative neoadjuvant therapy (including transarterial chemoembolization, radiotherapy, sorafenib, lenvatinib, immune checkpoint inhibitors, etc), (2) had primary HCC and underwent potential curative liver resection (macroscopically tumor-free), (3) diagnoses of HCC were confirmed by postoperative histopathology, and (4) suffered from chronic hepatitis $\mathrm{B}(\mathrm{CHB})$ or MAFLD. Patients were excluded if they were with (1) intrahepatic cholangiocarcinoma, (2) positive antiHCV or human immunodeficiency virus, (3) alcohol consumption, (4) autoimmune hepatitis, (5) drug-induced liver injury, (6) combined with other malignancies, or (7) incomplete medical information. All the procedures were carried out in accordance with the Helsinki Declaration of 1975. Written informed consent was not provided by the patients because this was a retrospective study. After obtaining institutional board review approval from Guangxi Medical Cancer Hospital (LW2021042) and the Fourth Affiliated Hospital of Guangxi Medical University (LW2021005), demographics, comorbid conditions, clinicopathologic data, radiology reports, and longterm outcomes of the included patients were reviewed.

Based on the aim of the study, included patients would be divided into three groups, namely, patients with MAFLD-related HCC (MAFLD group), patients with CHB-related HCC (CHB group), and those with dual etiology related HCC (CHB/ MAFLD group).

\section{Liver Resection}

The indications of liver resection were based on the Chinese guideline for HCC (18). Patients should be with Child-Pugh score of no more than 7 . The presence of appropriate residual liver volume was determined by volumetric dynamic enhanced computerized tomography or magnetic resonance imaging (MRI). The selection criteria and procedures of liver resection have been detailed elsewhere (19-21). The gallbladder was routinely excised before liver resection. Adequate drainage was routinely monitored. Intraoperative ultrasound was performed when necessary.

\section{Definition}

The diagnosis of MAFLD was based on hepatic steatosis [detected either by imaging techniques (ultrasound, computerized tomography or MRI) or by postoperative liver histopathology] in addition to overweight/obesity (body mass index $\geq 23 \mathrm{~kg} / \mathrm{m}^{2}$ ), presence of type 2 diabetes mellitus, or evidence of metabolic dysregulation $(10,11)$. Hepatic steatosis and cirrhosis were determined directly by imaging or histopathology reports. Fibrosis-4, waist circumference, insulin resistance score, plasma high-sensitivity C-reactive protein level, or blood biomarkers/ scores of MAFLD was not assessed in this study. Alcohol consumption was defined as excessive alcohol intake ( $>30 \mathrm{~g} /$ day in men, $>20 \mathrm{~g} /$ day in women) (22). In this study, dual etiology of 
HCC was defined as a patient suffered from both $\mathrm{CHB}$ and MAFLD (23). CHB was defined as the presence of positive of hepatitis B surface antigen HBV DNA, and/or hepatitis B core antibody. CHB-related HCC was defined as a patient with HCC accompanied by $\mathrm{CHB}$. Information on alcohol consumption, autoimmune hepatitis, and drug-induced liver injury was obtained from the past medical history or personal history of medical records. Major hepatectomy was defined as the resection of three or more Couinaud segments (24).

\section{Data Collection and Outcomes}

The number or level of the following data were collected and analyzed via the electronic medical records: gender, age, body mass index, hypertension, type 2 diabetes mellitus, hepatic steatosis, liver cirrhosis, Child-Pugh liver function grade, tumor size, tumor number, macrovascular invasion, Barcelona Clinical Liver Cancer stage, hepatitis B surface antigen, alpha fetoprotein, alanine aminotransferase, aspartate aminotransferase, plasma triglycerides, high density lipoprotein cholesterol, total bilirubin, albumin, prealbumin, microvascular invasion, resection type, and adjuvant transarterial chemoembolization.

The primary outcome was overall survival, calculated from the date of liver resection to the date of death from any cause or the date of the last follow-up. No patient undergone live transplantation in this cohort. Follow-up data of overall survival was obtained from the hospital database (X.-M.L). The secondary endpoints included recurrence-free survival and perioperative mortality and morbidity. Recurrence-free survival was calculated from the date of liver resection to the date of tumor recurrence or death from any cause, whichever occurred first. HCC recurrence, which was assessed by the investigators (J.-H.Z, Y.-X.T, K.C), was diagnosed using enhanced computed tomography and/or MRI [including gadolinium-ethoxybenzyl-diethylenetriamine pentaacetic acid (Gd-EOB-DTPA)-enhanced MRI] with or without alpha fetoprotein $\geq 400 \mathrm{ng} / \mathrm{mL}$ ) (18). Patients with HCC recurrence would be received appropriate therapeutic approaches, with radiofrequency ablation and repeat liver resection as the preferred curative treatments $(25,26)$. Postoperative mortality was defined as the rate of death within 30 days after liver resection. Perioperative morbidity was graded according to the ClavienDindo classification (27). Clavien-Dindo grade of at least 3a was defined as major morbidity. The follow-up period was up to March 2021 or death. Patients with $\mathrm{CHB}$ routinely received antiviral therapy with nucleos(t)ide analogue (28). Moreover, patients with high risk factors of HCC recurrence, such as tumor size at least $5 \mathrm{~cm}$, multinodules, involving macrovascular invasion or microvascular invasion, would receive adjuvant transarterial chemoembolization $(29,30)$. However, patients with MAFLD did not receive corresponding treatment for MAFLD.

\section{Statistical Analysis}

Categorical variables were expressed as number and percentage and compared with Pearson's chi-square tests. Bonferroni test was used for multiple comparisons. Continuous variables were expressed as mean \pm standard deviation and compared by using the one-way analysis of variance for normal distributions. For skewed distributions, variables were expressed as median (interquartile range) and compared by using the Kruskal-Wallis test. Overall and recurrence-free survival were calculated with the Kaplan-Meier method, with the values compared using the logrank test. To reveal the association between different counterparts (MAFLD, CHB/MAFLD, and CHB) and overall or recurrence-free survival after liver resection, multivariate analyses were performed using a Cox proportional hazards model. Any confounders that a change in effect estimate was more than $5 \%$ or recognized to be associated with the outcomes were entered into a Cox regression analysis. A two-tailed $p$ value $<0.05$ was considered to indicate statistically significant. GraphPad Prism 8.0 and IBM SPSS (ver. 26.0 SPSS Inc., Chicago, IL, USA) softwares were used to perform the analyses.

To reduce the potential effect of baseline variable imbalance on prognosis, propensity score matching between groups at a 1:3 ratio was used. All collected variables were involved in propensity score matching except body mass index, hypertension, type 2 diabetes mellitus, triglycerides, and high density lipoprotein cholesterol, which were used to define MAFLD. The propensity score was generated by a logistic regression. Nearest-neighbor caliper matching without replacement (random order or closest distance) was used to pair MAFLD (or MAFLD plus CHB/MAFLD) and $\mathrm{CHB}$ patients with similar propensity score values (31).

\section{RESULTS}

\section{Patient Demographic and Baseline Clinical Characteristics}

A total of 1887 patients underwent potential curative liver resection of HCC from January 2014 from December 2018; 368 had with anti-HCV or HIV positive, alcohol consumption, combined with other malignancies, autoimmune hepatitis or drug-induced liver injury and were excluded from this study. Other 194 patients without CHB or MAFLD were also excluded. Of the remaining 1325 patients, 67 (5.0\%) had MAFLD alone, 1082 (81.7\%) had CHB alone, and 176 (13.3\%) had CHB concurrent with MAFLD (Figure 1). The diagnosis of hepatic steatosis was detected by imaging techniques alone $(13,3.8 \%)$, liver histopathology alone $(299,88.2 \%)$ or confirmed by both $(27,8.0 \%)$.

Compared to patients with CHB, MAFLD patients were older (58.8 vs 48.9 yrs), more frequently had overweight/obesity $(62.7 \%$ vs $33.3 \%$ ), hypertension (35.8\% vs 6.4\%), type 2 diabetes mellitus (26.9\% vs $5.8 \%)$, and early stage disease (77.6\% vs $59.0 \%$, BCLC stage 0/A). Hepatic synthetic function at HCC presentation (measured by prothrombin time, prealbumin, alanine aminotransferase, and aspartate aminotransferase levels) was better in MAFLD patients. There were no differences in rates of gender, smoking, Child-Pugh grade B, or macrovascular invasion. Moreover, fewer MAFLD patients had cirrhosis (50.7\% vs $71.3 \%$ ), preoperative alpha fetoprotein concentration $\geq 400 \mathrm{ng} / \mathrm{mL}(23.9 \%$ vs $42.2 \%$ ), tumor size $\geq 5 \mathrm{~cm}(49.3 \%$ vs $61.6 \%)$, mulinodular 


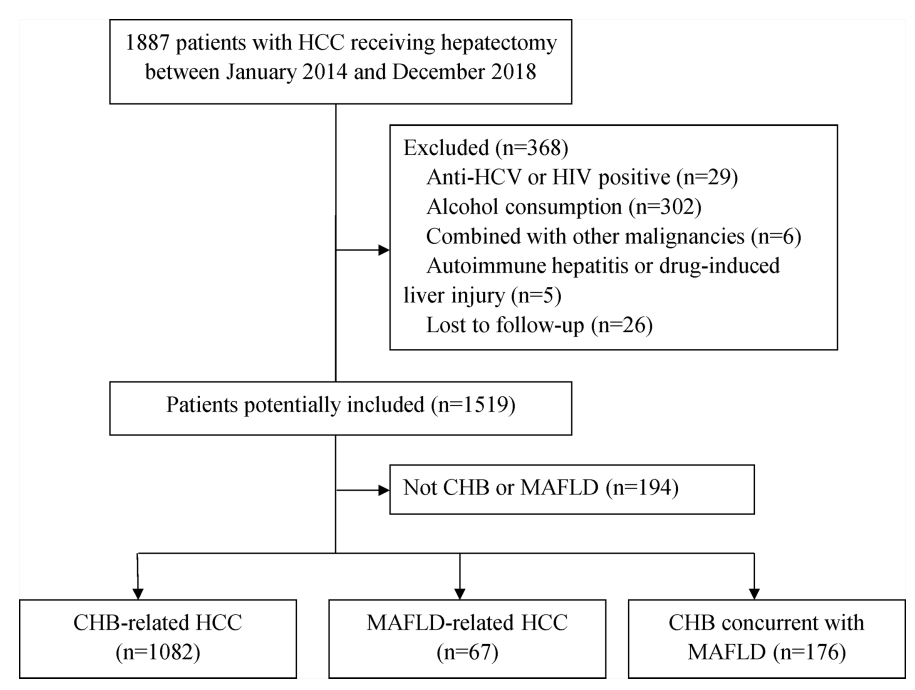

FIGURE 1 | Patient selection flow. CHB, chronic hepatitis B; HCC, hepatocellular carcinoma; HCV, hepatitis C virus; HIV, human immunodeficiency virus; MAFLD, metabolic dysfunction-associated fatty liver disease.

(10.4\% vs $22.5 \%$ ), microvascular invasion ( $41.8 \%$ vs $56.7 \%)$, and receiving major hepatectomy $(19.4 \%$ vs $34.1 \%)$ or adjuvant transarterial chemoembolization $(32.8 \%$ vs $55.0 \%)$ than $\mathrm{CHB}$ patients. However, MAFLD patients had higher level of triglycerides (Table 1).

\section{Survival Outcomes for Total Population}

During a median follow-up of 47 months, 450 of 1325 (34.0\%) patients died mainly because of HCC progression and/or hepatic failure. Of the total population $(\mathrm{n}=1325), 1$-year, 3-year, and 5year overall survival were $87.4 \%, 68.6 \%$, and $59.8 \%$, respectively (Supplementary Figure 1A). One-year, 3-year, and 5-year recurrence-free survival were $57.8 \%, 39.0 \%$, and $30.7 \%$, respectively (Supplementary Figure 1B). Univariable and multivariable analyses for overall and recurrence-free survival are summarized in Table 2. MAFLD was independently associated with overall and recurrence-free survival (all $P<0.05$ ) on multivariable analyses.

Postoperative mortality within 30 days after liver resection was observed in two $(0.2 \%)$ patients in $\mathrm{CHB}$ group. However, no postoperative mortality was observed in other two groups. MAFLD group $(4.5 \%, 3 / 67)$ had the lowest major morbidity than $\mathrm{CHB}$ group $(7.9 \%, 85 / 1082)$ and $\mathrm{CHB} / \mathrm{MAFLD}$ group (10.2\%, 18/176) (all $P>0.05)$.

Median follow-up for patients in the MAFLD, CHB/MAFLD, and $\mathrm{CHB}$ group were 46 (range, 7-87), 44 (range, 2-86), and 47 (range, 1-86) months, respectively. MAFLD patients had significantly higher overall and recurrence-free survival than CHB/MAFLD patients $(P=0.002$, Figure 2A; $P=0.038$, Figure 2B) or $\mathrm{CHB}$ patients $(P<0.001$, Figure 2A; $P<0.001$, Figure 2B). In addition, $C H B / M A F L D$ patients had higher overall ( $P=0.059$; Figure 2A) and recurrence-free survival than CHB patients $(P=0.010$; Figure 2B).

\section{Survival Outcomes Between MAFLD and CHB Patients After Propensity Score Matching}

Baseline clinical characteristics between MAFLD $(n=58)$ and CHB ( $\mathrm{n}=180)$ groups were comparable after propensity score matching (Table 3). MAFLD patients had significantly higher overall and recurrence-free survival than $\mathrm{CHB}$ patients $(P=0.004$, Figure 3A; $P=0.043$, Figure 3B).

\section{Survival Outcomes Between MAFLD Plus CHB/MAFLD and CHB Patients}

To further explore the difference in prognosis between MAFLD and $\mathrm{CHB}$, patients with MAFLD or CHB/MAFLD were combined in one group. Baseline clinical characteristics of the two groups before and after propensity matching were described in Table 4. Patients with MAFLD or CHB/MAFLD had significantly higher overall and recurrence-free survival than $\mathrm{CHB}$ patients before or after propensity score matching (all $P<0.05$, Figure 4).

\section{DISCUSSION}

MAFLD increased substantially over the past 20 years $(32,33)$. Moreover, concurrent diabetes mellitus, overweight/obesity, or hepatic steatosis is associated with increased HCC risk among $\mathrm{CHB}$ or $\mathrm{HCV}$ patients (34-37). These factors contribute to MAFLD becoming a major cause of HCC in the world. Our understanding of the prognoses of patients with MAFLD-related HCC after liver resection is limited. In agreement with other report (38), MAFLD patients more often had metabolic syndrome, had better synthetic liver function, less often had alpha fetoprotein concentration $\geq 400 \mathrm{ng} / \mathrm{mL}$, tumor size $\geq 5 \mathrm{~cm}$, multinodular, underwent major hepatectomy, and microvascular invasion compared to $\mathrm{CHB}$ counterparts at HCC diagnosis. 
TABLE 1 | Patient baseline demographic and clinical characteristics.

\begin{tabular}{|c|c|c|c|c|c|c|}
\hline \multirow[t]{2}{*}{ Variables } & \multirow{2}{*}{$\begin{array}{l}\text { MAFLD, } \\
\mathrm{n}=67(\%)\end{array}$} & \multirow{2}{*}{$\begin{array}{c}\text { CHB/MAFLD, } \\
n=176(\%)\end{array}$} & \multirow{2}{*}{$\begin{array}{c}\text { CHB, } \\
n=1082(\%)\end{array}$} & \multicolumn{3}{|c|}{$P$ value } \\
\hline & & & & $\begin{array}{l}\text { MAFLD vs } \\
\text { CHB }\end{array}$ & $\begin{array}{l}\text { MAFLD vs CHB/ } \\
\text { MAFLD }\end{array}$ & $\begin{array}{c}\text { CHB/MAFLD vs } \\
\text { CHB }\end{array}$ \\
\hline Gender, female & $10(14.9)$ & $21(11.9)$ & $151(14.0)$ & 0.824 & 0.532 & 0.469 \\
\hline Age, year & $58.8 \pm 10.0$ & $49.4 \pm 9.4$ & $48.9 \pm 10.8$ & $<0.001$ & $<0.001$ & 0.537 \\
\hline Body mass index, $\geq 23 \mathrm{~kg} / \mathrm{m}^{2}$ & $42(62.7)$ & $166(94.3)$ & $360(33.3)$ & $<0.001$ & $<0.001$ & $<0.001$ \\
\hline Smoking, present & $20(29.9)$ & $56(31.8)$ & $408(37.7)$ & 0.197 & 0.768 & 0.133 \\
\hline Hypertension, present & $24(35.8)$ & $29(16.5)$ & $69(6.4)$ & $<0.001$ & 0.001 & $<0.001$ \\
\hline Type 2 diabetes mellitus, present & $18(26.9)$ & $30(17.0)$ & $63(5.8)$ & $<0.001$ & 0.086 & $<0.001$ \\
\hline Hepatic steatosis, present & $67(100)$ & $176(100)$ & $96(8.9)$ & $<0.001$ & 1.000 & $<0.001$ \\
\hline Liver cirrhosis, present & $34(50.7)$ & $120(68.2)$ & $771(71.3)$ & $<0.001$ & 0.012 & 0.405 \\
\hline Child-Pugh grade B & $4(6.0)$ & $5(2.8)$ & $83(7.7)$ & 0.610 & 0.439 & 0.020 \\
\hline Triglycerides, $\mathrm{mmol} / \mathrm{L}$ & $1.3(0.9,1.8)$ & $0.9(0.9,1.4)$ & $0.9(0.7,1.1)$ & $<0.001$ & 0.041 & $<0.001$ \\
\hline High-density lipoprotein, mmol/L & $1.2 \pm 0.4$ & $1.2 \pm 0.3$ & $1.21 \pm 0.34$ & 0.314 & 0.317 & 0.941 \\
\hline Alpha fetoprotein, $\geq 400 \mathrm{ng} / \mathrm{ml}$ & $16(23.9)$ & $51(29.0)$ & 457 (42.2) & 0.003 & 0.427 & 0.001 \\
\hline Platelet count, $<100 \times 10^{9} / \mathrm{L}$ & $1(1.5)$ & $8(4.5)$ & $88(8.1)$ & 0.048 & 0.456 & 0.096 \\
\hline Prothrombin time & $12.2 \pm 0.9$ & $12.8 \pm 1.3$ & $12.9 \pm 1.4$ & $<0.001$ & $<0.001$ & 0.085 \\
\hline Total bilirubin, $\mu \mathrm{mol} / \mathrm{L}$ & $13.4(9.9,18.4)$ & $13.8(10.6,17.4)$ & $13.6(10.1,18.3)$ & 0.846 & 0.793 & 0.925 \\
\hline Albumin, $g / L$ & $39.6 \pm 4.2$ & $39.6 \pm 4.1$ & $38.9 \pm 4.7$ & 0.220 & 0.969 & 0.048 \\
\hline Prealbumin, g/L & $221.3 \pm 66.5$ & $195.2 \pm 57.5$ & $172.9 \pm 66.3$ & $<0.001$ & 0.003 & $<0.001$ \\
\hline Alanine aminotransferase, U/L & $26.0(18.5,35.0)$ & $40.0(27.0,56.0)$ & $37.0(26.0,54.0)$ & $<0.001$ & $<0.001$ & 0.122 \\
\hline Aspartate aminotransferase, U/L & $28.0(23.0,36.5)$ & $37.0(30.0,50.0)$ & $41.0(31.0,61.0)$ & $<0.001$ & $<0.001$ & 0.006 \\
\hline Tumor size, $>5 \mathrm{~cm}$ & $33(49.3)$ & $96(54.5)$ & $667(61.6)$ & 0.044 & 0.460 & 0.074 \\
\hline Tumor number, multiple & 7 (10.4) & $44(25.0)$ & $243(22.5)$ & 0.021 & 0.013 & 0.456 \\
\hline Macrovascular invasion, present & $7(10.4)$ & $21(11.9)$ & $197(18.2)$ & 0.107 & 0.746 & 0.041 \\
\hline BCLC stage & & & & 0.010 & 0.013 & 0.008 \\
\hline O/A & $52(77.6)$ & $106(60.2)$ & $638(59.0)$ & & & \\
\hline $\mathrm{B}$ & $6(9.0)$ & $45(25.6)$ & $196(18.1)$ & & & \\
\hline $\mathrm{C}$ & $9(13.4)$ & $25(14.2)$ & $248(22.9)$ & & & \\
\hline Major hepatectomy & $13(19.4)$ & $47(26.7)$ & $369(34.1)$ & 0.013 & 0.238 & 0.053 \\
\hline Microvascular invasion, present & $28(41.8)$ & $82(46.6)$ & $614(56.7)$ & 0.017 & 0.502 & 0.012 \\
\hline $\begin{array}{l}\text { Adjuvant transarterial } \\
\text { chemoembolization, present }\end{array}$ & $22(32.8)$ & $81(46.0)$ & $595(55.0)$ & $<0.001$ & 0.063 & 0.027 \\
\hline
\end{tabular}

Data are mean \pm standard deviation, median (IQR) or N (\%).

BCLC, Barcelona Clinic Liver Cancer; CHB, chronic hepatitis B; MAFLD, metabolic dysfunction-associated fatty liver disease.

These discrepancies in demographics, comorbidities, and measures of hepatic synthetic function may lead to lower perioperative major morbidity, but higher overall and recurrence-free survival compared to $\mathrm{CHB}$ counterparts. MAFLD patients still had a survival advantage after propensity score matching. Moreover, multivariate analyses confirmed the association between MAFLD and overall or recurrence-free survival independent of other clinicopathologic factors.

Among the patients with MAFLD-related HCC, 49.3\% were without cirrhosis. This finding reinforces the fact that MAFLDor NAFLD-related HCC could arise in the absence of cirrhosis in patients with clinically MAFLD or NAFLD (39-42). This phenomenon highlights the importance of HCC screening or surveillance programs for MAFLD or NAFLD patients without cirrhosis. However, the proportion of cirrhosis among the MAFLD patients with HCC was significantly lower than $\mathrm{CHB}$ or CHB/MAFLD counterparts, which is consistent with the findings that NAFLD patients with HCC had the lowest proportion of cirrhosis than those with any other etiologies including $\mathrm{CHB}, \mathrm{HCV}$ and alcoholic liver disease in Eastern or Western centers $(3,38,39,41,43-46)$. Hence, active HCC surveillance is recommended in MAFLD patients for the early detection of HCC.
Though the low proportion of cirrhosis, major hepatectomy, and better hepatic synthetic function at HCC presentation may translate into lower major morbidity and higher long-term survival after liver resection before propensity matching, MAFLD (or MAFLD plus CHB/MAFLD) patients still had significantly higher overall and recurrence-free survival than $\mathrm{CHB}$ patients after propensity matching. Therefore, other essential factor, such as the difference of pathophysiology between MAFLD and CHB $(47,48)$, may be at the root of the difference in prognoses between the groups. Future studies are expected to compare the difference in prognosis between groups from the perspective of pathophysiology.

MAFLD was originally known as NAFLD. In our cohort, the incidence of MAFLD was $12.9 \%(243 / 1887)$ - similar to that found in other series $(41,45)$. However, its incidence was higher in series from Korea (52.3\%) (46) or USA $(33.4 \%)$ (44). Though patients with concomitant nonalcoholic steatohepatitis and $\mathrm{CHB}$ have more advanced fibrosis, higher HCC risk, and shorter time to development of liver-related outcomes or death compared to patients with $\mathrm{CHB}$ alone (35, $37,49,50$ ), we did not found CHB/MAFLD patients had moresevere background liver disease or more advanced HCC than $\mathrm{CHB}$ patients. On the contrary, CHB/MAFLD counterparts had 


Univariable analysis

Multivariable analysis

Univariable analysis

Multivariable analysis

Hazard ratio $(95 \% \mathrm{Cl}) \quad P$ value $\quad$ Hazard ratio $(95 \% \mathrm{Cl}) \quad P$ value

Hazard ratio $(95 \% \mathrm{Cl})$

$P$ value

Hazard ratio $(95 \% \mathrm{CI})$

$P$ value

Counterparts

$\mathrm{CHB}$

MAFLD

1.00

$0.24(0.11-0.50)$

1.00

CHB/MAFL

$0.76(0.57-1.01)$

$<0.001$

$0.38(0.18-0.80)$

1.00

$0.44(0.29-0.68)$

$0.74(0.58-0.93)$

$<0.001$

1.00

Gender

Female

Age, yr

BMl, $\mathrm{kg} / \mathrm{m} 2$

$<23$

$\geq 23$

Smoking

Absent
Present

Hypertension

Absent

Present

T2DM

Absent

Hepatic steatosis

Absent

Present

Liver cirrhosis

Absent

1.00

$0.76(0.57-1.02)$

0.061

0.555

0.010

$0.54(0.35-0.83)$

0.005

1.00

$0.78(0.62-0.98)$

0.033

$0.99(0.98-1.00)$

0.002

1.00

1.00

1.00

$0.85(0.73-0.98)$

0.029

1.00

$0.73(0.61-0.89) \quad 0.002$

1.00

$1.21(1.00-1.46)$

0.047

1.00

1.00

$1.05(0.86-1.27)$

0.643

$1.21(1.04-1.41)$

0.014

$1.00(0.85-1.19)$

0.968

1.00

$0.86(0.62-1.20)$

0.371

1.00

$0.67(0.50-0.89)$

0.006

1.00
$0.81(0.57-1.14)$

$0.81(0.57-1.14$

0.227

$0.88(0.66-1.17)$

0.384

1.00

$0.68(0.54-0.85)$

1.00

$0.71(0.59-0.85)$

$<0.001$

Child-Pugh grade

A

1.00

$0.90(0.74-1.10)$

0.297

1.00

$0.94(0.80-1.10)$

0.452

1.00

$1.38(0.98-1.94) \quad 0.065$

$\begin{array}{lll}\text { Triglycerides, } \mathrm{mmol} / \mathrm{L} & 0.82(0.68-0.99) & 0.035\end{array}$

$\begin{array}{lll}\mathrm{HDL}, \mathrm{mmol} / \mathrm{L} & 0.77(0.58-1.04) & 0.087\end{array}$

Alpha fetoprotein, $\mathrm{ng} / \mathrm{ml}$

$<400$

$\geq 400$

0.087

1.00

$1.67(1.38-2.00)$

$<0.001$

1.00

Platelet count, $\times 10^{9} / \mathrm{L}$

$<100$

Prothrombin time

Total bilirubin, $\mu \mathrm{mol} / \mathrm{L}$

Albumin, $g / L$

Prealbumin, $g / L$

1.00

$0.73(0.52-1.01)$

0.060

$1.14(1.06-1.22)$

$<0.001$

$1.00(1.00-1.01)$

0.004

$0.99(0.99-1.00)$

$<0.001$

$1.00(1.00-1.00)$

$<0.001$

$\mathrm{ALT}, \mathrm{U} / \mathrm{L}$

$1.00(1.00-1.01)$

$<0.001$

$1.00(0.99-1.00)$

1.00

$1.18(0.87-1.60) \quad 0.285$

$0.86(0.74-0.99) \quad 0.034$

$1.08(0.86-1.37) \quad 0.498$

1.00

$1.73(1.49-2.00)$

$<0.001$

1.00

$$
1.00
$$

$0.91(0.69-1.21)$

$1.11(1.05-1.17)$

0.531

$\begin{array}{ll}1.11(1.05-1.17) & 0.336 \\ 0.97(0.95-0.98) & <0.001\end{array}$

$1.00(0.99-1.00)$

$1.00(1.00-1.00)$

$1.01(1.00-1.01)$

0.050
$<0.001$

$1.00(1.00-1.00)$ $1.01(1.00-1.01)$

0.038 


\begin{tabular}{|c|c|c|c|c|c|c|c|c|}
\hline \multirow[t]{3}{*}{ Variables } & \multicolumn{4}{|c|}{ Overall survival } & \multicolumn{4}{|c|}{ Recurrence-free survival } \\
\hline & \multicolumn{2}{|c|}{ Univariable analysis } & \multicolumn{2}{|c|}{ Multivariable analysis } & \multicolumn{2}{|c|}{ Univariable analysis } & \multicolumn{2}{|c|}{ Multivariable analysis } \\
\hline & Hazard ratio $(95 \% \mathrm{Cl})$ & $P$ value & Hazard ratio $(95 \% \mathrm{Cl})$ & $P$ value & Hazard ratio $(95 \% \mathrm{Cl})$ & $P$ value & Hazard ratio $(95 \% \mathrm{Cl})$ & $P$ value \\
\hline \multicolumn{9}{|c|}{ Tumor size, cm } \\
\hline$\leq 5$ & 1.00 & & 1.00 & & 1.00 & & 1.00 & \\
\hline$>5$ & $2.61(2.10-3.24)$ & $<0.001$ & $1.41(1.11-1.80)$ & 0.005 & $2.18(1.85-2.56)$ & $<0.001$ & $1.30(1.08-1.56)$ & 0.005 \\
\hline \multicolumn{9}{|c|}{ Tumor number } \\
\hline Single & 1.00 & & 1.00 & & 1.00 & & 1.00 & \\
\hline Multiple & $1.44(1.17-1.77)$ & 0.001 & $1.01(0.77-1.32)$ & 0.949 & $1.45(1.22-1.71)$ & $<0.001$ & $1.05(0.85-1.31)$ & 0.629 \\
\hline \multicolumn{9}{|l|}{ Resection } \\
\hline Minor & 1.00 & & 1.00 & & 1.00 & & 1.00 & \\
\hline Major & $2.23(1.85-2.69)$ & $<0.001$ & $1.22(0.99-1.51)$ & 0.060 & $1.86(1.60-2.16)$ & $<0.001$ & $1.02(0.86-1.21)$ & 0.825 \\
\hline \multicolumn{9}{|c|}{ Macrovascular invasion } \\
\hline Absent & 1.00 & & 1.00 & & 1.00 & & 1.00 & \\
\hline Present & 3.26 (2.66-3.99) & $<0.001$ & $1.57(1.07-2.28)$ & 0.019 & $2.66(2.23-3.17)$ & $<0.001$ & $1.31(0.96-1.79)$ & 0.083 \\
\hline \multicolumn{9}{|c|}{ Microvascular invasion } \\
\hline Absent & 1.00 & & 1.00 & & 1.00 & & 1.00 & \\
\hline Present & $2.85(2.32-3.51)$ & $<0.001$ & $2.14(1.72-2.66)$ & $<0.001$ & $2.16(1.84-2.52)$ & $<0.001$ & $1.54(1.30-1.81)$ & $<0.001$ \\
\hline \multicolumn{9}{|c|}{ Adjuvant TACE } \\
\hline No & 1.00 & & 1.00 & & 1.00 & & 1.00 & \\
\hline Yes & $1.69(1.40-2.06)$ & $<0.001$ & $1.17(0.96-1.43)$ & 0.125 & 1.99 (1.70-2.32) & $<0.001$ & $1.43(1.21-1.69)$ & $<0.001$ \\
\hline
\end{tabular}

ALT, Alanine aminotransferase; AST, aspartate aminotransferase; BMI, body mass index; CHB, chronic hepatitis B; HDL, High-density lipoprotein; MAFLD, metabolic dysfunction-associated fatty liver disease; T2DM, type 2 diabetes mellitus; TACE, transarterial chemoembolization. 

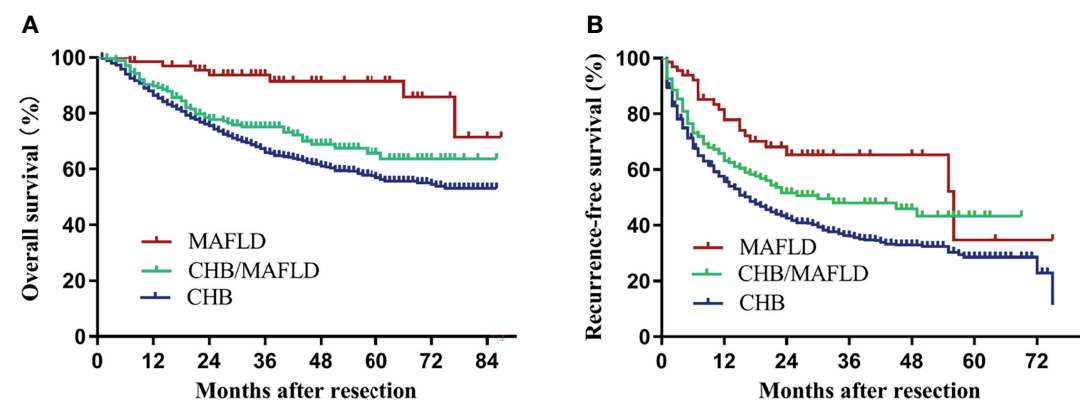

FIGURE 2 | Kaplan-Meier analysis of overall and recurrence-free survival for each counterparts. (A) Overall survival (MAFLD vs CHB, P<0.001; MAFLD vs CHB/ MAFLD, $P=0.002$; CHB/MAFLD vs CHB, $P=0.059$ ), (B) recurrence-free survival (MAFLD vs CHB, $P<0.001$; MAFLD vs CHB/MAFLD, $P=0.038$; CHB/MAFLD vs CHB, $P=0.010)$. CHB, chronic hepatitis B; MAFLD, metabolic dysfunction-associated fatty liver disease.

TABLE 3 | Baseline demographic and clinical characteristics of patients with MAFLD or CHB.

\begin{tabular}{|c|c|c|c|c|c|c|}
\hline \multirow[t]{2}{*}{ Variables } & \multicolumn{3}{|c|}{ Before propensity score } & \multicolumn{3}{|c|}{ After propensity score } \\
\hline & MAFLD, n=67 (\%) & CHB, $n=1082(\%)$ & $P$ value & MAFLD, $\mathrm{n}=58(\%)$ & CHB, $n=180(\%)$ & $P$ value \\
\hline Gender, female & $10(14.9)$ & $151(14.0)$ & 0.824 & $8(13,8)$ & $25(13.9)$ & 1.000 \\
\hline Age, year & $60(52,66)$ & $49(41,56)$ & $<0.001$ & $57(50,64)$ & $58(50,64)$ & 0.527 \\
\hline Smoking, present & $20(29.9)$ & $408(37.7)$ & 0.197 & $19(32.8)$ & $72(40.0)$ & 0.354 \\
\hline Liver cirrhosis, present & $34(50.7)$ & 771 (71.3) & $<0.001$ & $34(58.6)$ & $106(58.9)$ & 1.000 \\
\hline Child-Pugh grade B & $4(6.0)$ & $83(7.7)$ & 0.610 & $4(6.9)$ & $16(8.9)$ & 0.789 \\
\hline Alpha fetoprotein, $\geq 400 \mathrm{ng} / \mathrm{ml}$ & $16(23.9)$ & $457(42.2)$ & 0.003 & $15(25.9)$ & $56(31.1)$ & 0.511 \\
\hline Platelet count, $<100 \times 10^{9} / \mathrm{L}$ & $1(1.5)$ & $88(8.1)$ & 0.048 & $1(1.7)$ & $5(2.8)$ & 1.000 \\
\hline Prothrombin time & $12.1(11.5,12.8)$ & $12.8(12.1,13.7)$ & $<0.001$ & $12.1(11.5,12.7)$ & $12.3(11.7,13.0)$ & 0.094 \\
\hline Total bilirubin, $\mu \mathrm{mol} / \mathrm{L}$ & $13.4(9.9,18.4)$ & $13.6(10.1,18.3)$ & 0.846 & $13.5(9.8,19.1)$ & $12.7(9.6,17.8)$ & 0.465 \\
\hline Albumin, $g / L$ & $39.6(37.0,42.5)$ & $38.8(35.8,42)$ & 0.104 & $39.2(36.5,41.5)$ & $39.3(35.2,42.8)$ & 0.905 \\
\hline Prealbumin, g/L & $222(183,257)$ & $171(130,213)$ & $<0.001$ & $213(175,252)$ & $198(148,238)$ & 0.051 \\
\hline Alanine aminotransferase, U/L & $26.0(18.5,35.0)$ & $37.0(26.0,54.0)$ & $<0.001$ & $28.0(18.8,38.3)$ & $30.5(23.0,42.0)$ & 0.077 \\
\hline Aspartate aminotransferase, U/L & $28.0(23.0,36.5)$ & $41.0(31.0,61.0)$ & $<0.001$ & $26.0(17.0,38.0)$ & $38.0(24.0,42.0)$ & 0.074 \\
\hline Tumor size, $>5 \mathrm{~cm}$ & $33(49.3)$ & $667(61.6)$ & 0.044 & $28(48.3)$ & $92(51.1)$ & 0.764 \\
\hline Tumor number, multiple & 7 (10.4) & $243(22.5)$ & 0.021 & $7(12.1)$ & $18(10.0)$ & 0.806 \\
\hline Macrovascular invasion, present & $7(10.4)$ & $197(18.2)$ & 0.107 & $7(12.1)$ & $26(14.4)$ & 0.674 \\
\hline BCLC stage & & & 0.010 & & & 0.792 \\
\hline O/A & $52(77.6)$ & $638(59.0)$ & & $43(74.1)$ & $125(69.4)$ & \\
\hline $\mathrm{B}$ & $6(9.0)$ & $196(18.1)$ & & $6(10.3)$ & $23(12.8)$ & \\
\hline $\mathrm{C}$ & $9(13.4)$ & $248(22.9)$ & & $9(15.5)$ & $32(17.8)$ & \\
\hline Major hepatectomy & $13(19.4)$ & $369(34.1)$ & 0.013 & $11(19.0)$ & 37 (20.6) & 0.853 \\
\hline Microvascular invasion, present & $28(41.8)$ & $614(56.7)$ & 0.017 & $25(43.1)$ & $95(52.8)$ & 0.228 \\
\hline Adjuvant transarterial chemoembolization, present & $22(32.8)$ & $595(55.0)$ & $<0.001$ & $22(37.9)$ & $80(44.4)$ & 0.446 \\
\hline
\end{tabular}

Data are mean \pm standard deviation, median (IQR) or $N(\%)$.

BCLC, Barcelona Clinic Liver Cancer; CHB, chronic hepatitis B; MAFLD, metabolic dysfunction-associated fatty liver disease.

lower rate of cirrhosis $(68.2 \%$ vs $71.3 \%)$, alpha fetoprotein concentration $\geq 400 \mathrm{ng} / \mathrm{mL}$ ( $29 \%$ vs $42.2 \%)$, Barcelona Clinical Liver Cancer stage C disease (14.2\% vs $22.9 \%)$, tumor size $\geq 5 \mathrm{~cm}$ (54.5\% vs $61.6 \%)$, microvascular invasion $(46.6 \%$ vs $56.7 \%)$, receiving major hepatectomy (26.7\% vs $34.1 \%)$, and better hepatic synthetic function (measured by albumin, prealbumin, and aspartate aminotransferase levels) than $\mathrm{CHB}$ counterparts (Table 1). A cohort from Korea also found concurrent NAFLD was associated with both better overall and recurrence-free survival in patients with CHB-related HCC than those without NAFLD before adjusting for baseline characteristics (51). However, this survival benefit of the concurrent NAFLD was not significant in multivariable Cox analysis or analysis after propensity score matching (51). In our study, MAFLD counterparts had the highest overall and recurrence-free survival than $\mathrm{CHB} / \mathrm{MAFLD}$ or $\mathrm{CHB}$ counterparts, which was consistent with the findings that NAFLD-related HCC had better long-term survival outcomes compared to non-NAFLD etiologies after liver resection or radiofrequency ablation $(36,38,41,44,45)$. Our findings was confirmed by propensity score analysis.

The present study has some limitations. First, only patients with HCC underwent potential curative liver resection were included. The clinical impact of MAFLD on the prognoses of HCC patients underwent other therapies, such as transarterial chemoembolization or liver transplantation, could not be 

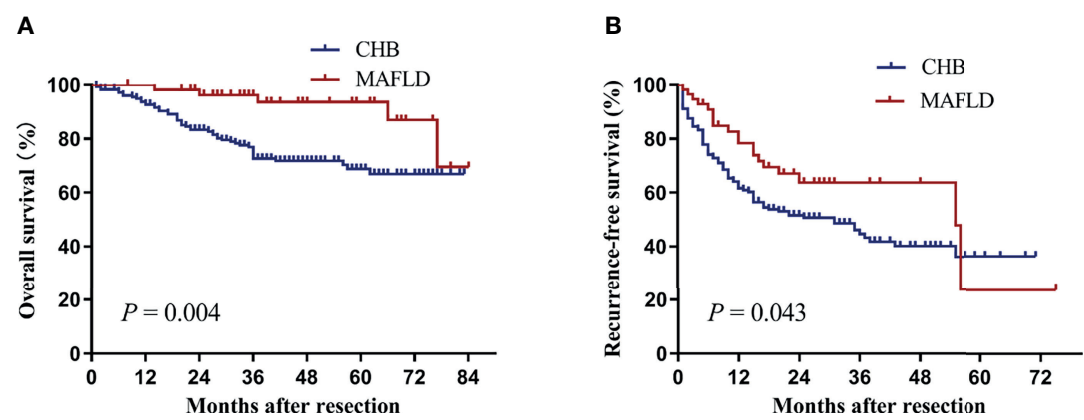

FIGURE 3 | Kaplan-Meier analysis of overall (A) and recurrence-free survival (B) for the MAFLD and CHB counterparts after propensity score matching. CHB, chronic hepatitis B; MAFLD, metabolic dysfunction-associated fatty liver disease.

TABLE 4 | Baseline demographic and clinical characteristics of patients with MAFLD+CHB/MAFLD or CHB.

\begin{tabular}{|c|c|c|c|c|c|c|}
\hline \multirow[t]{2}{*}{ Variables } & \multicolumn{3}{|c|}{ Before propensity score } & \multicolumn{3}{|c|}{ After propensity score } \\
\hline & $\begin{array}{l}\text { MAFLD or CHB/MAFLD, } \\
\qquad n=243(\%)\end{array}$ & $\begin{array}{c}\text { CHB, } n=1082 \\
(\%)\end{array}$ & $\begin{array}{c}P \\
\text { value }\end{array}$ & $\begin{array}{l}\text { MAFLD or CHB/MAFLD, } \\
\qquad \mathrm{n}=232(\%)\end{array}$ & $\begin{array}{c}\mathrm{CHB}, \mathrm{n}=698 \\
(\%)\end{array}$ & $\begin{array}{c}P \\
\text { value }\end{array}$ \\
\hline Gender, female & $31(12.8)$ & $151(14.0)$ & 0.681 & $30(12.9)$ & $100(14.3)$ & 0.662 \\
\hline Age, year & $51(44,60)$ & $49(41,56)$ & $<0.001$ & $50(44,59)$ & $50(42,58)$ & 0.207 \\
\hline Smoking, present & 76 (31.3) & $408(37.7)$ & 0.065 & $76(32.8)$ & $246(35.2)$ & 0.524 \\
\hline Liver cirrhosis, present & $154(71.3)$ & $771(71.3)$ & 0.017 & $152(65.5)$ & $489(70.1)$ & 0.219 \\
\hline Child-Pugh grade B & $9(3.7)$ & $83(7.7)$ & 0.035 & $9(3.9)$ & $37(5.3)$ & 0.485 \\
\hline Alpha fetoprotein, $\geq 400 \mathrm{ng} / \mathrm{ml}$ & $67(27.6)$ & 457 (42.2) & $<0.001$ & $67(28.9)$ & $234(33.5)$ & 0.196 \\
\hline Platelet count, $<100 \times 10^{9} / \mathrm{L}$ & $9(3.7)$ & $88(8.1)$ & 0.020 & $9(3.9)$ & $40(5.7)$ & 0.313 \\
\hline Prothrombin time & $12.4(11.7,13.3)$ & $\begin{array}{c}12.8(12.1 \\
13.7)\end{array}$ & $<0.001$ & $12.5(11.7,13.3)$ & $\begin{array}{c}12.6(12.0 \\
13.4)\end{array}$ & 0.132 \\
\hline Total bilirubin, $\mu \mathrm{mol} / \mathrm{L}$ & $13.8(10.3,17.6)$ & $\begin{array}{c}13.6(10.1 \\
18.3)\end{array}$ & 0.986 & $13.8(10.2,17.8)$ & $13.2(9.9,17.6)$ & 0.339 \\
\hline Albumin, g/L & $39.7(36.8,42.4)$ & $\begin{array}{c}38.8(35.8 \\
42.0)\end{array}$ & 0.007 & $39.6(36.6,42.2)$ & $\begin{array}{c}39.2(36.2 \\
42.1)\end{array}$ & 0.223 \\
\hline Prealbumin, g/L & $202(163,239)$ & $171(130,213)$ & $<0.001$ & $197(162,236)$ & $190(150,230)$ & 0.077 \\
\hline Alanine aminotransferase, U/L & $35(25,52)$ & $37(26,54)$ & 0.253 & $36(25,53)$ & $34(25,51)$ & 0.611 \\
\hline Aspartate aminotransferase, $U / L$ & $35(27,48)$ & $41(31,61)$ & $<0.001$ & $35(27,48)$ & $37(29,52)$ & 0.067 \\
\hline Tumor size, $>5 \mathrm{~cm}$ & $129(53.1)$ & $667(61.6)$ & 0.017 & 125 (53.9) & 407 (58.3) & 0.251 \\
\hline Tumor number, multiple & $51(21.0)$ & $243(22.5)$ & 0.670 & $50(21.6)$ & $145(20.8)$ & 0.852 \\
\hline Macrovascular invasion, present & $28(11.5)$ & $197(18.2)$ & 0.014 & $28(12.1)$ & $102(14.6)$ & 0.382 \\
\hline BCLC stage & & & 0.008 & & & 0.193 \\
\hline $\mathrm{O} / \mathrm{A}$ & $158(65.0)$ & $638(59.0)$ & & $148(63.8)$ & 435 (62.3) & \\
\hline $\mathrm{B}$ & $51(21.0)$ & $196(18.1)$ & & $50(21.6)$ & $127(18.2)$ & \\
\hline $\mathrm{C}$ & $34(14.0)$ & $248(22.9)$ & & $34(14.7)$ & $136(19.5)$ & \\
\hline Major hepatectomy & $60(24.7)$ & $369(34.1)$ & 0.005 & $58(25.0)$ & $207(29.7)$ & 0.180 \\
\hline Microvascular invasion, present & $110(45.3)$ & $614(56.7)$ & 0.001 & $107(46.1)$ & $351(50.3)$ & 0.289 \\
\hline $\begin{array}{l}\text { Adjuvant transarterial chemoembolization, } \\
\text { present }\end{array}$ & $103(42.4)$ & $595(55.0)$ & $<0.001$ & $102(44.0)$ & 339 (48.6) & 0.226 \\
\hline
\end{tabular}

Data are mean \pm standard deviation, median (IQR) or N (\%).

BCLC, Barcelona Clinic Liver Cancer; CHB, chronic hepatitis B; MAFLD, metabolic dysfunction-associated fatty liver disease.

evaluated in this study. Second, mild hepatic steatosis may not be detectable by imaging techniques. And fibroscan was not routinely used in the present cohort. Moreover, some cases with hepatic steatosis may not be documented in postoperative histopathological report. As a result, the actual number of MAFLD in the enrolled population may be more than that in the reported population. Third, patients with $\mathrm{CHB}$ were included in two groups. Antiviral therapy with nucleos(t)ide analogue would improve overall survival (28). Nevertheless, MAFLD patients still had the best long-term survival compared to $\mathrm{CHB}$ or CHB/MAFLD counterparts. Finally, subgroup analysis based on tumor stage or alpha fetoprotein concentration was not performed because of the small sample size of MAFLD group.

\section{IN CONCLUSION}

Patients with HCC in the setting of MAFLD have less-severe background liver disease and better long-term outcomes after 

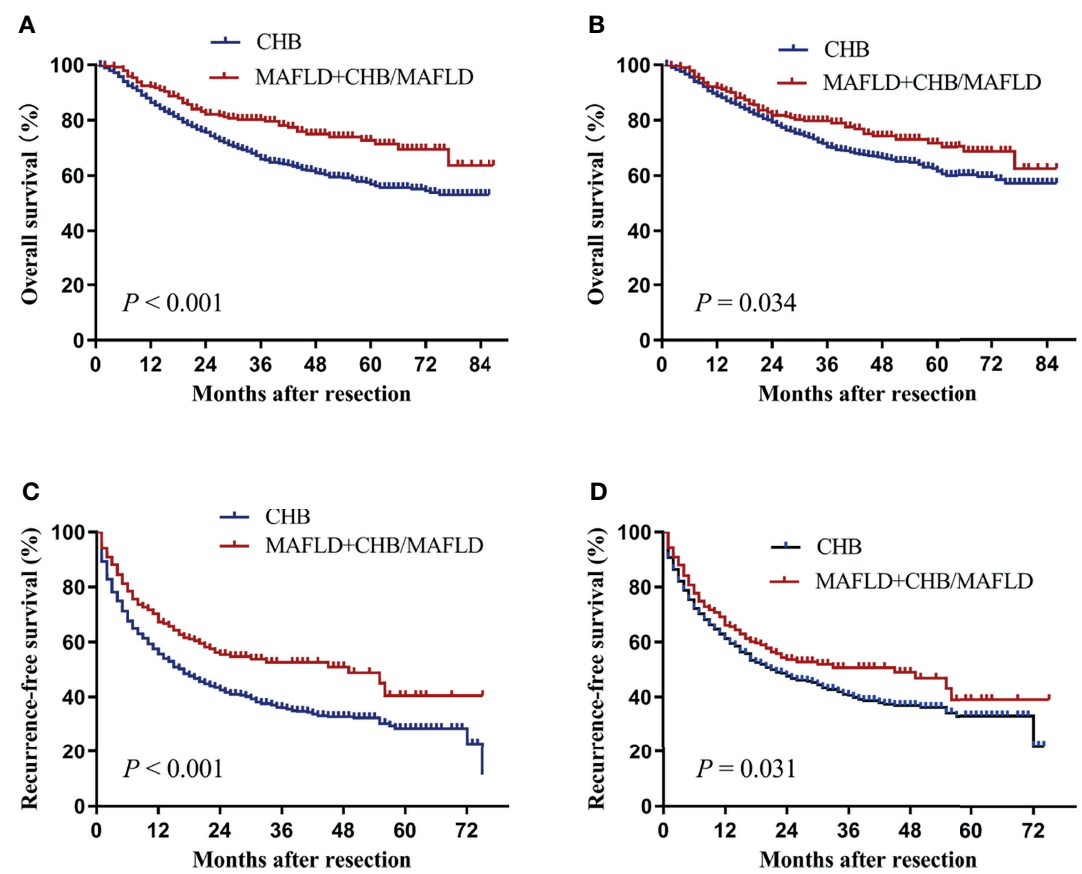

FIGURE 4 | Kaplan-Meier analysis of overall and recurrence-free survival for the MAFLD plus CHB/MAFLD and CHB counterparts. (A) Overall survival before propensity matching, (B) overall survival after propensity matching, (C) recurrence-free survival before propensity matching, (D) recurrence-free survival after propensity matching. $\mathrm{CHB}$, chronic hepatitis $\mathrm{B}$; MAFLD, metabolic dysfunction-associated fatty liver disease.

potential curative liver resection compared to counterparts with CHB. However, the association between MAFLD and HCC patient prognoses was not fully assessed because the relatively small number of MAFLD patients examined and the potential selection bias. Further well-designed study with a larger sample size is warranted.

\section{DATA AVAILABILITY STATEMENT}

The datasets presented in this article are not readily available because all data were in the manuscript. Supplementary Material for this article is available by request to J-HZ. Requests to access the datasets should be directed to J-HZ, zhongjianhong@gxmu.edu.cn.

\section{ETHICS STATEMENT}

This study was approved by the Ethics Committees of our hospitals (LW2021042; LW2021005). Written informed consent for participation was not required for this study in accordance with the national legislation and the institutional requirements.

\section{AUTHOR CONTRIBUTIONS}

J-HZ conceived the study. All authors participated in the acquisition of the data. R-RH and J-HZ analyzed data. LL and
J-HZ drafted and revised the manuscript. All authors contributed to the article and approved the submitted version.

\section{FUNDING}

J-HZ is in part supported by the National Natural Science Foundation of China (82060510), 'Guangxi BaGui Scholars' Special Fund (2019AQ20), the Natural Science Foundation of Guangxi Province (2020GXNSFAA159022), and the Guangxi Undergraduate Training Program for Innovation and Entrepreneurship (202110598178, 202110598073). B-DX is in part supported by the "High-level innovation team and outstanding scholar program in Guangxi Colleges and Universities". X-ML is in part supported by the Self-raised Scientific Research Fund of the Ministry of Health of Guangxi Province (Z20200923).

\section{SUPPLEMENTARY MATERIAL}

The Supplementary Material for this article can be found online at: https://www.frontiersin.org/articles/10.3389/fonc.2021.783339/ full\#supplementary-material

Supplementary Figure 1 | Kaplan-Meier analysis of overall (A) and recurrencefree survival (B) for the total population. 


\section{REFERENCES}

1. Sung H, Ferlay J, Siegel RL, Laversanne M, Soerjomataram I, Jemal A, et al. Global Cancer Statistics 2020: GLOBOCAN Estimates of Incidence and Mortality Worldwide for 36 Cancers in 185 Countries. CA Cancer J Clin (2021) 71(3):209-49. doi: 10.3322/caac.21660

2. Llovet JM, Kelley RK, Villanueva A, Singal AG, Pikarsky E, Roayaie S, et al. Hepatocellular Carcinoma. Nat Rev Dis Primers (2021) 7(1):6. doi: 10.1038/ s41572-020-00240-3

3. Pais R, Fartoux L, Goumard C, Scatton O, Wendum D, Rosmorduc O, et al. Temporal Trends, Clinical Patterns and Outcomes of NAFLD-Related HCC in Patients Undergoing Liver Resection Over a 20-Year Period. Aliment Pharmacol Ther (2017) 46(9):856-63. doi: 10.1111/apt.14261

4. Younossi ZM, Otgonsuren M, Henry L, Venkatesan C, Mishra A, Erario M, et al. Association of Nonalcoholic Fatty Liver Disease (NAFLD) With Hepatocellular Carcinoma (HCC) in the United States From 2004 to 2009. Hepatology (2015) 62(6):1723-30. doi: 10.1002/hep.28123

5. Eguchi Y, Wong G, Lee IH, Akhtar O, Lopes R, Sumida Y. Hepatocellular Carcinoma and Other Complications of Non-Alcoholic Fatty Liver Disease and Non-Alcoholic Steatohepatitis in Japan: A Structured Review of Published Works. Hepatol Res (2021) 51(1):19-30. doi: 10.1111/hepr.13583

6. Huang DQ, El-Serag HB, Loomba R. Global Epidemiology of NAFLD-Related HCC: Trends, Predictions, Risk Factors and Prevention. Nat Rev Gastroenterol Hepatol (2021) 18(4):223-38. doi: 10.1038/s41575-020-00381-6

7. Younossi Z, Anstee QM, Marietti M, Hardy T, Henry L, Eslam M, et al. Global Burden of NAFLD and NASH: Trends, Predictions, Risk Factors and Prevention. Nat Rev Gastroenterol Hepatol (2018) 15(1):11-20. doi: 10.1038/nrgastro.2017.109

8. European Association for the Study of the Liver (EASL), European Association for the Study of Diabetes (EASD) and European Association for the Study of Obesity (EASO). EASL-EASD-EASO Clinical Practice Guidelines for the Management of Non-Alcoholic Fatty Liver Disease. J Hepatol (2016) 64(6):1388-402. doi: 10.1016/j.jhep.2015.11.004

9. Chalasani N, Younossi Z, Lavine JE, Charlton M, Cusi K, Rinella M, et al. The Diagnosis and Management of Nonalcoholic Fatty Liver Disease: Practice Guidance From the American Association for the Study of Liver Diseases. Hepatology (2018) 67(1):328-57. doi: 10.1002/hep.29367

10. Eslam M, Newsome PN, Sarin SK, Anstee QM, Targher G, Romero-Gomez M, et al. A New Definition for Metabolic Dysfunction-Associated Fatty Liver Disease: An International Expert Consensus Statement. J Hepatol (2020) 73 (1):202-9. doi: 10.1016/j.jhep.2020.07.045

11. Eslam M, Sarin SK, Wong VW, Fan JG, Kawaguchi T, Ahn SH, et al. The Asian Pacific Association for the Study of the Liver Clinical Practice Guidelines for the Diagnosis and Management of Metabolic Associated Fatty Liver Disease. Hepatol Int (2020) 14(6):889-919. doi: 10.1007/s12072020-10094-2

12. Shiha G, Alswat K, Al Khatry M, Sharara AI, Örmeci N, Waked I, et al. Nomenclature and Definition of Metabolic-Associated Fatty Liver Disease: A Consensus From the Middle East and North Africa. Lancet Gastroenterol Hepatol (2021) 6(1):57-64. doi: 10.1016/S2468-1253(20)30213-2

13. Lim S, Kim JW, Targher G. Links Between Metabolic Syndrome and Metabolic Dysfunction-Associated Fatty Liver Disease. Trends Endocrinol Metab (2021) 32(7):500-14. doi: 10.1016/j.tem.2021.04.008

14. Myers S, Neyroud-Caspar I, Spahr L, Gkouvatsos K, Fournier E, Giostra E, et al. NAFLD and MAFLD as Emerging Causes of HCC: A Populational Study. JHEP Rep (2021) 3(2):100231. doi: 10.1016/j.jhepr.2021.100231

15. European Association for the Study of the Liver. EASL Clinical Practice Guidelines: Management of Hepatocellular Carcinoma. J Hepatol (2018) 69 (1):182-236. doi: 10.1016/j.jhep.2011.12.001

16. Zhong JH, Ke Y, Wang YY, Li LQ. Liver Resection for Patients With Hepatocellular Carcinoma and Macrovascular Invasion, Multiple Tumours, or Portal Hypertension. Gut (2015) 64(3):520-1. doi: 10.1136/gutjnl-2014-308139

17. Lim KC, Chow PK, Allen JC, Siddiqui FJ, Chan ES, Tan SB. Systematic Review of Outcomes of Liver Resection for Early Hepatocellular Carcinoma Within the Milan Criteria. Br J Surg (2012) 99(12):1622-9. doi: 10.1002/bjs.8915

18. Zhou J, Sun H, Wang Z, Cong W, Wang J, Zeng M, et al. Guidelines for the Diagnosis and Treatment of Hepatocellular Carcinoma (2019 Edition). Liver Cancer (2020) 9(6):682-720. doi: 10.1159/000509424
19. Zhong JH, Xiang BD, Gong WF, Ke Y, Mo QG, Ma L, et al. Comparison of Long-Term Survival of Patients With BCLC Stage B Hepatocellular Carcinoma After Liver Resection or Transarterial Chemoembolization. PloS One (2013) 8(7):e68193. doi: 10.1371/journal.pone.0068193

20. Zhong JH, Ke Y, Gong WF, Xiang BD, Ma L, Ye XP, et al. Hepatic Resection Associated With Good Survival for Selected Patients With Intermediate and Advanced-Stage Hepatocellular Carcinoma. Ann Surg (2014) 260(2):329-40. doi: 10.1097/SLA.0000000000000236

21. Li C, Chen K, Liu X, Liu HT, Liang XM, Liang GL, et al. Analysis of Clinicopathological Characteristics and Prognosis of Young Patients With Hepatocellular Carcinoma After Hepatectomy. J Clin Transl Hepatol (2020) 8 (3):285-91. doi: 10.14218/JCTH.2020.00021

22. Arab JP, Dirchwolf M, Alvares-da-Silva MR, Barrera F, Benítez C, Castellanos-Fernandez M, et al. Latin American Association for the Study of the Liver (ALEH) Practice Guidance for the Diagnosis and Treatment of Non-Alcoholic Fatty Liver Disease. Ann Hepatol (2020) 19(6):674-90. doi: 10.1016/j.aohep.2020.09.006

23. Boyle M, Masson S, Anstee QM. The Bidirectional Impacts of Alcohol Consumption and the Metabolic Syndrome: Cofactors for Progressive Fatty Liver Disease. J Hepatol (2018) 68(2):251-67. doi: 10.1016/j.jhep.2017.11.006

24. Pol B, Campan P, Hardwigsen J, Botti G, Pons J, Le Treut YP. Morbidity of Major Hepatic Resections: A 100-Case Prospective Study. Eur J Surg (1999) 165(5):446-53. doi: 10.1080/110241599750006686

25. Zhong JH, Xing BC, Zhang WG, Chan AW, Chong CCN, Serenari M, et al. Repeat Hepatic Resection Versus Radiofrequency Ablation for Recurrent Hepatocellular Carcinoma: A Retrospective Multicentre Study. Br J Surg (2021) 109(1):71-8. doi: 10.1093/bjs/znab1340

26. Xing H, Sun LY, Yan WT, Quan B, Liang L, Li C, et al. Repeat Hepatectomy for Patients With Early and Late Recurrence of Hepatocellular Carcinoma: A Multicenter Propensity Score Matching Analysis. Surgery (2021) 169(4):91120. doi: 10.1016/j.surg.2019.11.005

27. Dindo D, Demartines N, Clavien PA. Classification of Surgical Complications: A New Proposal With Evaluation in a Cohort of 6336 Patients and Results of a Survey. Ann Surg (2004) 240(2):205-13. doi: 10.1097/01.sla.0000133083.54934.ae

28. Li C, Li ZC, Ma L, Li LQ, Zhong JH, Xiang BD, et al. Perioperative Antiviral Therapy Improves the Prognosis of HBV DNA-Negative Patients With HBVRelated Hepatocellular Carcinoma. Expert Rev Gastroenterol Hepatol (2020) 14(8):749-56. doi: 10.1080/17474124.2020.1784727

29. Zhong JH, Li LQ. Postoperative Adjuvant Transarterial Chemoembolization for Participants With Hepatocellular Carcinoma: A Meta-Analysis. Hepatol Res (2010) 40(10):943-53. doi: 10.1111/j.1872-034X.2010.00710.x

30. Qi YP, Zhong JH, Liang ZY, Zhang J, Chen B, Chen CZ, et al. Adjuvant Transarterial Chemoembolization for Patients With Hepatocellular Carcinoma Involving Microvascular Invasion. Am J Surg (2019) 217 (4):739-44. doi: 10.1016/j.amjsurg.2018.07.054

31. McCaffrey DF, Griffin BA, Almirall D, Slaughter ME, Ramchand R, Burgette LF. A Tutorial on Propensity Score Estimation for Multiple Treatments Using Generalized Boosted Models. Stat Med (2013) 32(19):3388-414. doi: 10.1002/ sim. 5753

32. Han MAT, Yu Q, Tafesh Z, Pyrsopoulos N. Diversity in NAFLD: A Review of Manifestations of Nonalcoholic Fatty Liver Disease in Different Ethnicities Globally. J Clin Transl Hepatol (2021) 9(1):71-80. doi: 10.14218/ JCTH.2020.00082

33. Kumar R, Priyadarshi RN, Anand U. Non-Alcoholic Fatty Liver Disease: Growing Burden, Adverse Outcomes and Associations. J Clin Transl Hepatol (2020) 8(1):76-86. doi: 10.14218/JCTH.2019.00051

34. Dyal HK, Aguilar M, Bartos G, Holt EW, Bhuket T, Liu B, et al. Diabetes Mellitus Increases Risk of Hepatocellular Carcinoma in Chronic Hepatitis C Virus Patients: A Systematic Review. Dig Dis Sci (2016) 61(2):636-45. doi: 10.1007/s10620-015-3983-3

35. Chan TT, Chan WK, Wong GL, Chan AW, Nik Mustapha NR, Chan SL, et al. Positive Hepatitis B Core Antibody Is Associated With Cirrhosis and Hepatocellular Carcinoma in Nonalcoholic Fatty Liver Disease. Am J Gastroenterol (2020) 115(6):867-75. doi: 10.14309/ajg.0000000000000588

36. Nishio T, Hatano E, Sakurai T, Taura K, Okuno M, Kasai Y, et al. Impact of Hepatic Steatosis on Disease-Free Survival in Patients With Non-B Non-C Hepatocellular Carcinoma Undergoing Hepatic Resection. Ann Surg Oncol (2015) 22(7):2226-34. doi: 10.1245/s10434-014-4181-9 
37. Chan AW, Wong GL, Chan HY, Tong JH, Yu YH, Choi PC, et al. Concurrent Fatty Liver Increases Risk of Hepatocellular Carcinoma Among Patients With Chronic Hepatitis B. J Gastroenterol Hepatol (2017) 32(3):667-76. doi: 10.1111/jgh.13536

38. Reddy SK, Steel JL, Chen HW, DeMateo DJ, Cardinal J, Behari J, et al. Outcomes of Curative Treatment for Hepatocellular Cancer in Nonalcoholic Steatohepatitis Versus Hepatitis C and Alcoholic Liver Disease. Hepatology (2012) 55(6):1809-19. doi: 10.1002/hep.25536

39. Piscaglia F, Svegliati-Baroni G, Barchetti A, Pecorelli A, Marinelli S, Tiribelli C, et al. Clinical Patterns of Hepatocellular Carcinoma in Nonalcoholic Fatty Liver Disease: A Multicenter Prospective Study. Hepatology (2016) 63(3):82738. doi: 10.1002/hep.28368

40. Kawada N, Imanaka K, Kawaguchi T, Tamai C, Ishihara R, Matsunaga T, et al. Hepatocellular Carcinoma Arising From Non-Cirrhotic Nonalcoholic Steatohepatitis. J Gastroenterol (2009) 44(12):1190-4. doi: 10.1007/s00535009-0112-0

41. Koh YX, Tan HJ, Liew YX, Syn N, Teo JY, Lee SY, et al. Liver Resection for Nonalcoholic Fatty Liver Disease-Associated Hepatocellular Carcinoma. J Am Coll Surg (2019) 229(5):467-78.e461. doi: 10.1016/j.jamcollsurg.2019.07.012

42. Lee TY, Wu JC, Yu SH, Lin JT, Wu MS, Wu CY. The Occurrence of Hepatocellular Carcinoma in Different Risk Stratifications of Clinically Noncirrhotic Nonalcoholic Fatty Liver Disease. Int J Cancer (2017) 141 (7):1307-14. doi: 10.1002/ijc.30784

43. Yang T, Hu LY, Li ZL, Liu K, Wu H, Xing H, et al. Liver Resection for Hepatocellular Carcinoma in Non-Alcoholic Fatty Liver Disease: A Multicenter Propensity Matching Analysis With HBV-HCC. J Gastrointest Surg (2020) 24(2):320-9. doi: 10.1007/s11605-018-04071-2

44. Wong CR, Njei B, Nguyen MH, Nguyen A, Lim JK. Survival After Treatment With Curative Intent for Hepatocellular Carcinoma Among Patients With vs Without Non-Alcoholic Fatty Liver Disease. Aliment Pharmacol Ther (2017) 46(11-12):1061-9. doi: 10.1111/apt.14342

45. Lin BZ, Lin TJ, Lin CL, Liao LY, Chang TA, Lu BJ, et al. Differentiation of Clinical Patterns and Survival Outcomes of Hepatocellular Carcinoma on Hepatitis B and Nonalcoholic Fatty Liver Disease. J Chin Med Assoc (2021) 84 (6):606-13. doi: 10.1097/JCMA.0000000000000530

46. Jung YB, Yoo JE, Han DH, Kim KS, Choi JS, Kim DY, et al. Clinical and Survival Outcomes After Hepatectomy in Patients With Non-Alcoholic Fatty Liver and Hepatitis B-Related Hepatocellular Carcinoma. HPB (Oxford) (2021) 23(7):1113-22. doi: 10.1016/j.hpb.2020.10.027
47. Li JZ, Ye LH, Wang DH, Zhang HC, Li TY, Liu ZQ, et al. The Identify Role and Molecular Mechanism of the MALAT1/hsa-Mir-20b-5p/TXNIP Axis in Liver Inflammation Caused by CHB in Patients With Chronic HBV Infection Complicated With NAFLD. Virus Res (2021) 298:198405. doi: 10.1016/ j.virusres.2021.198405

48. Zhu YF, Wang J, Fang JZ, Yang Q, Lv FF. Interface Hepatitis Over Grade 2 May Differentiate Chronic Inflammation Associated With CHB From NAFLD in the Early Stage. Gastroenterol Res Pract (2020) 2020:3584568. doi: $10.1155 / 2020 / 3584568$

49. Choi HSJ, Brouwer WP, Zanjir WMR, de Man RA, Feld JJ, Hansen BE, et al. Nonalcoholic Steatohepatitis Is Associated With Liver-Related Outcomes and All-Cause Mortality in Chronic Hepatitis B. Hepatology (2020) 71(2):539-48. doi: 10.1002/hep.30857

50. Lee YB, Ha Y, Chon YE, Kim MN, Lee JH, Park H, et al. Association Between Hepatic Steatosis and the Development of Hepatocellular Carcinoma in Patients With Chronic Hepatitis B. Clin Mol Hepatol (2019) 25(1):52-64. doi: $10.3350 / \mathrm{cmh} .2018 .0040$

51. Yoon JS, Lee HY, Chung SW, Kim SW, Chang Y, Lee YB, et al. Prognostic Impact of Concurrent Nonalcoholic Fatty Liver Disease in Patients With Chronic Hepatitis B-Related Hepatocellular Carcinoma. J Gastroenterol Hepatol (2020) 35(11):1960-8. doi: 10.1111/jgh.15026

Conflict of Interest: The authors declare that the research was conducted in the absence of any commercial or financial relationships that could be construed as a potential conflict of interest.

Publisher's Note: All claims expressed in this article are solely those of the authors and do not necessarily represent those of their affiliated organizations, or those of the publisher, the editors and the reviewers. Any product that may be evaluated in this article, or claim that may be made by its manufacturer, is not guaranteed or endorsed by the publisher.

Copyright (C) 2022 Liu, Xie, Teng, Deng, Chen, Liu, Huo, Liang, Guo, Yang, Ma, Xiang, Li and Zhong. This is an open-access article distributed under the terms of the Creative Commons Attribution License (CC BY). The use, distribution or reproduction in other forums is permitted, provided the original author(s) and the copyright owner(s) are credited and that the original publication in this journal is cited, in accordance with accepted academic practice. No use, distribution or reproduction is permitted which does not comply with these terms. 